\title{
Numerical-analytic method for implicit differential equations
}

\author{
Tadeusz Jankowski
}




\title{
NUMERICAL-ANALYTIC METHOD FOR IMPLICIT DIFFERENTIAL EQUATIONS
}

\author{
TADEUSZ JANKOWSKI \\ Department of Differential Equations, Technical University of Gdańsk \\ 11/12 Narutowicz Str., 80-152 Gdańsk, Poland \\ tjany@mif.pg.gda.pl
}

[Received May 14, 2001]

\begin{abstract}
The numerical-analytic method combined with the comparison one based on successive approximations is used to investigate solutions of implicit differential equations with integral boundary conditions. Implicit systems of the neutral type with deviated arguments are also considered.
\end{abstract}

Mathematical Subject Classification: 34B95

Keywords: Numerical-analytic method, integral boundary condition, convergence of approximate solutions, implicit problems, deviated arguments

\section{Introduction}

A useful approach to studying the existence of solutions of boundary-value problems is the numerical-analytic method, for details see [6], [11] and the references there. Recently it was extended in several directions to different problems, see for example [5]-[14]. In this paper we apply this technique to the implicit differential system of the form

$$
F\left(t, x(t), x^{\prime}(t)\right)=\Theta, \quad t \in J=[0, T]
$$

with the integral boundary condition

$$
A_{0} x(0)+\int_{0}^{T} D(s) x(s) d s+A_{1} x(T)=d,
$$

where $F \in C\left(J \times R^{p} \times R^{p}, R^{p}\right)$, and $\Theta$ is zero vector in $R^{p}$. In the above, the matrices $\left(A_{0}\right)_{p \times p},\left(A_{1}\right)_{p \times p}, D_{p \times p}$ and $d_{p \times 1}$ are given. Furthermore we assume that the matrix $D$ is continuous.

The numerical-analytic method is used to formulate corresponding existence results for problems of type (1.1)-(1.2) under the assumption that $F$ satisfies the Lipschitz condition with respect to the second variable and the extra one with respect to the last variable in matrix notation. This method combined with the comparison one offers the convergence of approximate solutions to the solution of (1.1)-(1.2). In our investigation, we discuss the sufficient conditions for such convergence and some results (containing also conditions on the spectral radius of corresponding matrices) 
are given. A more general implicit problem with deviated arguments of neutral type is also considered and corresponding existence results are formulated.

\section{Assumptions}

Put

$$
\begin{aligned}
& \mathcal{L}(t, x)=\left(1-\frac{t}{T}\right) \int_{0}^{t} x(s) d s-\frac{t}{T} \int_{t}^{T} x(s) d s \\
& D_{0}=\int_{5}^{T} D(s) d s, \quad D_{1}=\int_{0}^{T} s D(s) d s \\
& D_{2}=\left(A_{7} T+D_{1}\right)^{-1}, \quad D_{3}\left(\bar{x}_{0}\right)=D_{2}\left[d-\left(A_{0}+A_{1}+D_{9}\right) \bar{x}_{1}\right]
\end{aligned}
$$

assuming that the matrix $D_{5}$ exists. According to the numerical-analytic method, we need to find a parameter $\alpha$ such that $x(t)=\bar{x}_{0}+\mathcal{L}(t, z)+\alpha t$ satisfies the boundary condition from (1.2). Then putting $x^{\prime}=z$, we obtain the following auxiliary system

$$
\mathcal{F}\left(t, \bar{x}_{0}, z, z\right)=\Theta, \quad t \in J,
$$

where

$$
\mathcal{F}\left(t, \bar{x}_{0}, u, v\right)=F\left(t, \bar{x}_{0}+\mathcal{L}(t, u)-t D_{2} \int_{0}^{T} D(s) \mathcal{L}(s, u) d s+t D_{3}\left(\bar{x}_{0}\right), v(t)\right), \quad t \in J
$$

Note that $x(9)=\bar{x}_{0}$. Indeed, a solution $z$ of (2.1) depends on $\bar{x}_{0}$.

Let us introduce the following

Assumption $H_{1}$. There are matrices $K_{p \times p}, L_{p \times p}$ with nonnegative entries, $L^{-1}$ exists, $L^{-1}$ has nonnegative entries and such that

$$
\begin{aligned}
& |F(t, x, y)-F(t, \bar{x}, y)| \leq K|x-\bar{x}|, \\
& |F(t, x, y)-F(t, x, \bar{y})| \geq L|y-\bar{y}|
\end{aligned}
$$

for all $t \in J, x, \bar{x}, y, \bar{y} \in R^{p}$. Here $|\cdot|$ denotes the absolute value of the vector, so $|x|=\left(\left|x_{1}\right|, \cdots,\left|x_{p}\right|\right)^{T}$.

Assumption $H_{2}$. For any nonnegative function $h \in C\left(J \times R^{p}, R_{+}^{p}\right)$ there exists a unique solution $u \in C\left(J, R_{+}^{p}\right)$ of the comparison equation

$$
\Gamma u(t)+h\left(t, \bar{x}_{0}\right)=u(t), \quad t \in J,
$$

where

$$
\begin{aligned}
& \Gamma u(t)=L^{-1} K\left[\Omega u(t)+\left|D_{2}\right| t \int_{1}^{T}|D(s)| \Omega u(s) d s\right], \quad t \in J, \\
& \Omega u(t)=\left(1-\frac{t}{T}\right) \int_{0}^{t} u(s) d s+\frac{t}{T} \int_{t}^{T} u(s) d s, \quad t \in J .
\end{aligned}
$$




\section{Lemmas}

For $t \in J, n=0,1, \cdots$, let us define the sequence $\left\{u_{n}\right\}$ by

$$
u_{n+1}(t)=\Gamma u_{n}(t), \quad u_{0}(t)=u(t)
$$

where $u$ is defined as in Assumption $H_{2}$ with $h\left(t, \bar{x}_{0}\right)=L^{-1}\left|\mathcal{F}\left(t, x_{0}, z_{0}, z_{0}\right)\right|$.

To obtain a solution of problem (2.1), we shall first establish some properties for the sequence $\left\{u_{n}\right\}$. They are given in the next two lemmas.

Lemma 1 Let Assumptions $\mathrm{H}_{1}$ and $\mathrm{H}_{2}$ be satisfied. Assume that the matrix $\mathrm{D}_{2}$ exists. Then

$$
u_{n+1}(t) \leq u_{n}(t) \leq u_{0}(t), \quad t \in J, n=0,1, \cdots,
$$

and the sequence $\left\{u_{n}\right\}$ converges uniformly to zero function, so $u_{n}(t) \rightarrow 0, t \in J$ if $n \rightarrow \infty$.

Proof. Note that $u_{1}(t)=\Gamma u_{1}(t) \leq u_{0}(t), t \in J$, by Assumption $H_{2}$. By induction in $n$, we are able to prove that $u_{o+1}(t) \leq u_{n}(t), \quad t \in J, \quad n=0,1, \cdots$ because operator $\Gamma$ is nondecreasing. Now, if $n \rightarrow \infty$, then $u_{n} \rightarrow u$, where $u$ is a solution of the equation $u(t)=\Gamma u(t), t \in J$. By Assumption $H_{2}, u(t)=0$ on $J$. The proof is complete.

Lemma 2 Assume that $F \in C\left(J \times R^{p} \times R^{p}, R^{p}\right)$, and $\left(A_{0}\right)_{p \times p},\left(A_{1}\right)_{p \times p}, D_{p \times p}$ and $d_{p \times 1}$ are given matrices and $D$ is continuous on $J$. Assume that the matrix $D_{2}$ exists. Let Assumptions $H_{1}$ and $H_{2}$ be satisfied. For any $v \in C\left(J, R^{p}\right)$ there is a solution $z$ of the equation $F\left(t, \bar{x}_{0}, v, z\right)=\Theta$. Then

$$
\left|z_{n+k}(t)-z_{k}(t)\right| \leq u_{k}(t), \quad t \in J, \quad n, k=0,1, \cdots,
$$

where $z_{0} \in C\left(J, R^{p}\right)$, and $\mathcal{F}\left(t, \bar{x}_{0}, z_{n}, z_{n+1}\right)=\Theta, \quad t \in J$.

Moreover, the functions $x_{n}$ of the form

$$
x_{n}(t)=x_{0}+\mathcal{L}\left(t, z_{n}\right)+t D_{3}\left(\bar{x}_{0}\right)-t D_{2} \int_{9}^{T} D(s) \mathcal{L}\left(s, z_{n}\right) d s=d, \quad t \in J
$$

satisfy boundary condition (1.2) for all $n=0,1, \cdots$.

Proof. Note that our method is implicit since for each $n$ we need to find the element $z_{n+1}$ by solving the equation $\mathcal{F}\left(t, \bar{x}_{0}, y_{n}, y\right)=\Theta$. This equation has a unique solution. To show this we assume that it has two different solutions $y$ and $\bar{y}$. Then the relation $\mathcal{F}\left(t, \bar{x}_{0}, y_{n}, y\right)-\mathcal{F}\left(t, \bar{x}_{0}, y_{n}, \bar{y}\right)=\Theta$ follows $|y(t)-\bar{y}(t)| \leq \Theta$, by Assumption $H_{1}$. It proves that $y=\bar{y}$ on $J$, so the sequence $\left\{y_{n}\right\}$ is well defined.

Now we need to show (3.1). Indeed,

$$
\left|z_{1}(t)-z_{0}(t)\right| \leq h\left(t, x_{0}\right) \leq u_{0}(t), \quad t \in J
$$


if we apply Assumption $H_{1}$ to the relation

$$
\mathcal{F}\left(t, \bar{x}_{0}, z_{0}, z_{1}\right)-\mathcal{F}\left(t, \bar{x}_{0}, z_{1}, z_{0}\right)=-\mathcal{F}\left(t, \bar{x}_{0}, z_{0}, z_{0}\right) .
$$

Assume that $\left|z_{k}(t)-z_{0}(t)\right| \leq u_{0}(t), \quad t \in J$ for $k \geq 0$. Then, using Assumption $H_{1}$ to the equality

$\mathcal{F}\left(t, \bar{x}_{0}, z_{k}, z_{k+1}\right)-\mathcal{F}\left(t, \bar{x}_{0}, z_{k}, z_{0}\right)=\mathcal{F}\left(t, \bar{x}_{0}, z_{0}, z_{0}\right)-\mathcal{F}\left(t, \bar{x}_{0}, z_{k}, z_{0}\right)-\mathcal{F}\left(t, \bar{x}_{0}, z_{0}, z_{0}\right)$

we have

$$
\begin{aligned}
\left|z_{k+1}(t)-z_{0}(t)\right| & \left.\leq L^{-1} K\left[\left|\mathcal{L}\left(t, z_{k}\right)-\mathcal{L}\left(t, z_{0}\right)\right|+t\left|D_{2}\right| \int_{0}^{T}|D(s)| \mid \mathcal{L}\left(s, z_{k}\right)-\mathcal{L}\left(s, z_{0}\right)\right] d s\right] \\
& +h\left(t, \bar{x}_{0}\right) \leq \Gamma u_{0}(t)+h\left(t, \bar{x}_{0}\right)=u_{0}(t), \quad t \in J .
\end{aligned}
$$

Hence, by mathematical induction, we have $\left|z_{n}(t)-z_{0}(t)\right| \leq u_{0}(t), \quad t \in J$ for $n=$ $0,1, \cdots$. Based on the above, let us assume that $\left|z_{n+k}(t)-z_{k}(t)\right| \leq u_{k}(t), \quad t \in J$ for all $n$ and some $k \geq 0$. Then, using again Assumption $H_{1}$ to the relation

$\mathcal{F}\left(t, \bar{x}_{0}, z_{n+k}, z_{n+k+1}\right)-\mathcal{F}\left(t, \bar{x}_{0}, z_{n+k}, z_{k+1}\right)=\mathcal{F}\left(t, \bar{x}_{0}, z_{k}, z_{k+1}\right)-\mathcal{F}\left(t, \bar{x}_{0}, z_{n+k}, z_{k+1}\right)$

we have

$$
\left|z_{n+k+1}(t)-z_{k+1}(t)\right| \leq L^{-1} K\left[\Omega u_{k}(t)+t\left|D_{2}\right| \int_{0}^{T}|D(s)| \Omega u_{k}(s) d s\right]=u_{k+1}(t)
$$

for $t \in J$. Hence, by mathematical induction, (3.1) holds. It ends to proof.

\section{Existence results}

Combining Lemmas 1 and 2 we have

Theorem 3 Let all assumptions of Lemma 2 be satisfied. Then, for every $\bar{x}_{0} \in R^{p}$, there exists a solution $\bar{z}$ of problem (2.1) where $z_{n}(t) \rightarrow \bar{z}(t), \quad t \in J$ as $n \rightarrow \infty$ and we have the estimate

$$
\left|z_{n}(t)-\bar{z}(t)\right| \leq u_{n}(t), \quad t \in J
$$
iff

Moreover, the function $x(t)=\bar{x}_{0}+\int_{0}^{t} \bar{z}(s) d s$ is the solution of problem (1.1)-(1.2)

$$
\frac{1}{T} \int_{0}^{T} \bar{z}(s) d s+D_{2} \int_{0}^{T} D(s) \mathcal{L}(s, \bar{z}) d s=D_{3}\left(\bar{x}_{0}\right) .
$$

Remark 4 Let matrix $\mathrm{D}_{2}$ exist. Assumption $\mathrm{H}_{2}$ is satisfied if

$$
\rho(Z)<1, \text { where } Z=L^{-1} K\left[I+\left|D_{2}\right| T \int_{0}^{T}|D(s)| d s\right] \frac{T}{2} .
$$


Here $\rho(Z)$ denotes the spectral radius of the matrix $Z$. To get condition (4.2) we need to apply the Banach fixed point theorem to equation (2.2). Denote the left-hand side of problem $(2.2)$ by $\Lambda$. Let $u, \bar{u} \in C\left(J, R_{+}^{p}\right)$. Then

$$
\begin{aligned}
|\Lambda u-\Lambda \bar{u}| & \leq L^{-1} K\left[|\Omega u(t)-\Omega \bar{u}(t)|+\left|D_{2}\right| t \int_{0}^{T}|D(s)||\Omega u(s)-\Omega \bar{u}(s)| d s\right] \\
& \leq Z \max _{t \in J}|u(t)-\bar{u}(t)| .
\end{aligned}
$$

Hence, operator $\Lambda$ is a contraction mapping, so problem (2.2) has a unique solution by the Banach fixed point theorem.

Remark 5 Indeed, condition $\rho(Z)<1$ holds if

$$
T\left\|L^{-1} K\right\|\left[1+\left\|D_{2}\right\| T \int_{0}^{T}\|D(s)\| d s\right]<2,
$$

where $\|\cdot\|$ denotes the maximum norm.

Remark 6 If $A_{0}=A_{1}=0_{p \times p}$, and $D(t)=I_{p \times p}, t \in J$, then $D_{2}=\frac{2}{T^{2}} I$, and then $Z=\frac{1}{2} T L^{-1} K$.

Remark 7 Let $D(t)=0_{p \times p}, t \in J$. Then, by Remark 4, Assumption $\mathrm{H}_{2}$ holds if $\rho\left(\frac{T}{2} L^{-1} K\right)<1$. Note that by [7], we have the weaker condition, namely $\rho\left(\frac{3}{10} T L^{-1} K\right)$ $<1$.

Remark 8 If $F(t, u, v)=f(t, u)-v$, then $L=I$, and we have the problem considered in [4]-[14].

\section{Implicit systems with deviated arguments of the neutral type}

Let $\alpha_{i}, \beta_{j} \in C(J, J), i=1,2, \cdots, r, j=1,2, \cdots, m$. Let us consider the following differential equation

$$
G\left(t, x\left(\alpha_{1}(t)\right), x\left(\alpha_{2}(t)\right), \cdots, x\left(\alpha_{r}(t)\right), x^{\prime}\left(\beta_{1}(t)\right), x^{\prime}\left(\beta_{2}(t)\right), \cdots, x^{\prime}\left(\beta_{m}(t)\right), x^{\prime}(t)\right)=\Theta
$$

for $t \in J$, where $G \in C\left(J \times\left(R^{p}\right)^{r+m+1}, R^{p}\right)$. Note that if $G$ is solvable with respect to the last variable, then we have the problem considered in [1], [3], see also [6], [9]. As in section 2, according to the numerical-analytic method, we have the following auxiliary problem

$$
\mathcal{G}\left(t, \bar{x}_{0}, z, z\right)=\Theta, \quad t \in J
$$

where

$$
\begin{aligned}
& \mathcal{G}\left(t, \bar{x}_{0}, u, v\right) \\
& =G\left(t, u_{1}\left(t ; \bar{x}_{0}\right), u_{2}\left(t ; \bar{x}_{0}\right), \cdots, u_{r}\left(t ; \bar{x}_{0}\right), u\left(\beta_{1}(t)\right), u\left(\beta_{2}(t)\right), \cdots, u\left(\beta_{m}(t)\right), v(t)\right),
\end{aligned}
$$


and for $i=1,2, \cdots, r$,

$$
u_{i}\left(t ; \bar{x}_{0}\right)=\bar{x}_{0}+\mathcal{L}\left(\alpha_{i}(t), u\right)-D_{2} \alpha_{i}(t) \int_{0}^{T} D(s) \mathcal{L}(s, u) d s+D_{3}\left(\bar{x}_{0}\right) \alpha_{i}(t), \quad t \in J
$$

Now, we introduce the following

Assumption $H_{3}$. There are matrices $K_{p \times p}^{i}, L_{p \times p}^{j}$ and $L_{p \times p}$ with nonnegative entries, $i=1,2, \cdots, r, \quad j=1,2, \cdots, m$ and such that $L^{-1}$ exists, $L^{-1}$ has nonnegative entries and

$$
\begin{aligned}
\mid G\left(t, x_{1}, x_{2}, \cdots, x_{r}, y_{1}, y_{2}, \cdots, y_{m}, y\right) & -G\left(t, \bar{x}_{1}, \bar{x}_{2}, \cdots, \bar{x}_{r}, \bar{y}_{1}, \bar{y}_{2}, \cdots, \bar{y}_{m}, y\right) \mid \\
\leq & \sum_{i=1}^{r} K^{i}\left|x_{i}-\bar{x}_{i}\right|+\sum_{j=1}^{m} L^{j}\left|y_{q}-\bar{y}_{j}\right|, \\
\mid G\left(t, x_{1}, x_{2}, \cdots, x_{r}, y_{1}, y_{2}, \cdots, y_{m}, y\right) & -G\left(t, x_{1}, x_{2}, \cdots, x_{r}, y_{1}, y_{2}, \cdots, y_{m}, \bar{y}\right)|\geq L| y-\bar{y} \mid
\end{aligned}
$$

for all $t \in J, x_{i}, \bar{x}_{i}, y_{j}, \bar{y}_{j}, y, \bar{y} \in R^{p}, i=1,2, \cdots, r, j=1,2, \cdots, m$.

Assumption $H_{4}$. For any nonnegative function $H \in C\left(J \times R^{p}, R_{+}^{p}\right)$ there exists a unique solution $v \in C\left(J, R_{+}^{p}\right)$ of the comparison equation

$$
\Gamma_{1} v(t)+H\left(t, \bar{x}_{0}\right)=v(t), \quad t \in J,
$$

where

$\Gamma_{1} v(t)=L^{-7} \sum_{i=1}^{r} K^{i}\left[\Omega v\left(\alpha_{i}(t)\right)+\left|D_{2}\right| \alpha_{i}(t) \int_{0}^{T}|D(s)| \Omega v(s) d s\right]+L^{-1} \sum_{j=1}^{m} L^{j} v\left(\beta_{j}(t)\right)$.

Let $y, \bar{y}, z \in C\left(J, R^{p}\right)$. Then, by Assumption $H_{3}$ we have

$$
\left|\mathcal{G}\left(t, \bar{x}_{0}, y, z\right)-\mathcal{G}\left(t, \bar{x}_{0}, \bar{y}, z\right)\right| \leq L \Gamma_{1} w(t) \quad \text { for } \quad w=|y-\bar{y}| .
$$

For $t \in J, n=0,1, \cdots$, let us define the sequence $\left\{v_{n}\right\}$ by

$$
v_{0}(t)=v(t), \quad v_{n+1}(t)=\Gamma_{1} v_{n}(t),
$$

where $v$ is defined as in Assumption $H_{4}$ with $H\left(t, \bar{x}_{0}\right)=L^{-1}\left|\mathcal{G}\left(t, \bar{x}_{0}, z_{0}, z_{0}\right)\right|$.

For $z_{0} \in C\left(J, R^{p}\right)$, we define the sequence $\left\{z_{n}\right\}$ by relations: $\mathcal{G}\left(t, \bar{x}_{0}, z_{n}, z_{n+1}\right)=\Theta$ for $t \in J$, and $n=0,1, \ldots$ Then we can formulate the following

Theorem 9 Assume that $\left.G \in C\left(J \times R^{p}\right)^{r+m+1}, R^{p}\right), \alpha_{i}, \beta_{j} \in C(J, J)$, and $\left(A_{0}\right)_{p \times p}$, $\left(A_{1}\right)_{p \times p}, D_{p \times p}$ and $d_{p \times 1}$ are given matrices and $D$ is continuous on $J$. Assume that matrix $D_{2}$ exists. Let Assumptions $\mathrm{H}_{3}$ and $C_{4}$ be satisfied. Assume that for any $u \in C(J, R)$ there is a solution $z$ of $\mathcal{G}\left(t, \bar{x}_{0}, u, z\right)=\Theta$. Then, for every $\bar{x}_{0} \in R^{p}$, the sequence $\left\{z_{n}\right\}$ converges to the solution $\bar{z}$ of problem (5.2), so $z_{n}(t) \rightarrow \bar{z}(t)$ for $t \in J$ if $n \rightarrow \infty$ and for $t \in J$ we have the error estimate

$$
\left|z_{n}(t)-\bar{z}(t)\right| \leq v_{n}(t), \quad t \in J, \quad n=0,1, \cdots .
$$


Moreover, the function $x(t)=\bar{x}_{0}+\int_{0}^{t} \bar{z}(s) d s$ is the solution of problem (5.1) with condition (1.2) iff relation (4.2) holds.

Proof. Note that the sequence $\left\{v_{n}\right\}$ converges to zero function (the proof is similar to the proof of Lemma 3). Using relation (5.4), by mathematical induction, we can show that $\left|z_{n+k}(t)-z_{k}(t)\right| \leq v_{k}(t), \quad t \in J, n, k=0,1, \cdots$ (the proof is similar to that of Lemma 2). The rest is obvious and the proof is finished.

Remark 10 Note that Assumption $H_{4}$ holds if it is assumed that

$2 \sum_{i=1}^{r}\left\|L^{-1} K^{i}\right\| \max _{t \in J} \alpha_{i}(t)\left[1-\frac{\alpha_{i}(t)}{T}\right]\left[1+\left\|D_{2}\right\| \alpha_{i}(t) \int_{0}^{T}\|D(s)\| d s\right]+\sum_{j=1}^{m}\left\|L^{-1} L^{j}\right\|<1$.

Remark 11 Let $m=1$ and $\beta_{1}(t)=t$. Then operator $\Gamma_{1}$ has the form

$$
\Gamma_{1} v(t)=\Gamma_{2} v(t)+A v(t) \quad \text { for } \quad A=L^{-1} L^{1}
$$

and

$$
\Gamma_{2} v(t)=L^{-1} \sum_{i=1}^{r} K^{i}\left[\Omega v\left(\alpha_{i}(t)\right)+\left|D_{2}\right| \alpha_{i}(t) \int_{0}^{T}|D(s)| \Omega v(s) d s\right] .
$$

Assume that $\rho(A)<1$, so the matrix $(I-A)^{-1}$ exists and its entries are nonnegativ. Then equation (1.1) takes the form

$$
(I-A)^{-1}\left[\Gamma_{2} v(t)+H\left(t, \bar{x}_{0}\right)\right]=v(t), \quad t \in J .
$$

In this case Assumption $H_{4}$ holds if $\rho(A)<1$ and $\rho(B)<1$ for

$$
B=2[L(I-A)]^{-1} \sum_{i=1}^{r} K^{i}\left[I+\left|D_{2}\right| T \int_{0}^{T}|D(s)| d s\right] \max _{t \in J} \alpha_{i}(t)\left[1-\frac{\alpha_{i}(t)}{T}\right] .
$$

\section{REFERENCES}

[1] Augustynowicz, A. And Kwapisz, M.: On a numerical-analytic method of solving of boundary value problem for functional differential equation of neutral type, Math. Nachr., 145 (1990), 255-269.

[2] Jankowski, T.: Numerical-analytic method for differential-algebraic systems, Acta Math. Hungar., to appear.

[3] KWAPISZ, M.: Some remarks on an integral equation arising in applications of numericalanalytic method of solving of boundary value problems, Ukrain. Mat. Zh., 44 (1992), $128-132$.

[4] Martynyuk, S.V.: Investigation of the solutions of differential equations with integral boundary conditions (in Russian), in Nonlinear problems in the theory of differential equations (in Russian), 59-65, Akad. Nauk Ukrain. SSR, Inst. Mat., Kiev, 1991. 
[5] MeLnik, Yu.I.: On a modification of a numerical-analytic method for solving two-point boundary value problems for ordinary differential equations (in Russian), Differ. Uravn., 32 (1996), 249-255.

[6] Ronto, M. and Samollenko, A.M.: Numerical-Analytic Methods in the Theory of Boundary-Value Problems, World Scientific, Singapore, 2000.

[7] Ronto, M. and Mesarosh, I.: Some remarks on the convergence of the numericalanalytic methods to successive approximations (in Russian), Ukrain. Mat. Zh., 48 (1996), 90-95.

[8] Ronto, N.I., Samoilenko, A.M. and S.I.Trofimchuk, S.I.: The theory of the numericalanalytic method: achevements and new directions of development, Ukrain. Math. J., 50 (1998), 116-135.

[9] Ronto, N.I., Samoilenko, A.M. and Trofimchuk, S.I.: Theory of the numericalanalytic method: achevements and new directions of development III, Ukrain. Math. J., 50 (1998), 960-979.

[10] Samollenko, A.M. and Ronto, N.I.: A numerical-analytic method for solving boundary value problems for ordinary differential equations (in Russian), Ukrain. Mat. Zh., 33 (1981), 467-475.

[11] Samoilenko, A.M. And Ronto, N.I.: Numerical-analytic methods of investigating boundary value problems (in Russian), Nauk. Dumka, Kiev, 1986.

[12] Samollenko, A.M. And Ronto, N.I.: A modification of numerical-analytic method of successive approximations for boundary value problems for ordinary differential equations (in Russian), Ukrain. Mat. Zh., 42 (1990), 1107-1116.

[13] Samoilenko, A.M., Ronto, N.I. and Martynyuk, S.V.: On the numerical-analytic method for problems with integral boundary conditions (in Russian), Dokl. Akad. Nauk Ukrain. SSR, 4 (1991), 34-37.

[14] Samollenko, A.M. and Martynyuk, S.V.: Justification of the numerical-analytic method of successive approximations for problems with integral boundary conditions (in Russian), Ukrain. Mat. Zh., 9 (1991), 1231-1239. 